\title{
Intermediate Grade Lung Neuroendocrine Neoplasm
}

National Cancer Institute

\section{Source}

National Cancer Institute. Intermediate Grade Lung Neuroendocrine Neoplasm. NCI

Thesaurus. Code C45568.

An intermediate grade malignant neoplasm with neuroendocrine differentiation that arises from the lung. This category includes atypical carcinoid tumor. 